\title{
Quantitative assessment of gadolinium deposition in dentate nucleus using quantitative susceptibility mapping
}

\section{$\operatorname{AUTHOR}(\mathrm{S}):$}

Hinoda, Takuya; Fushimi, Yasutaka; Okada, Tomohisa; Arakawa, Yoshiki; Liu, Chunlei; Yamamoto, Akira; Okada, Tsutomu; Yoshida, Kazumichi; Miyamoto, Susumu; Togashi, Kaori

\section{CITATION:}

Hinoda, Takuya ... [et al]. Quantitative assessment of gadolinium deposition in dentate nucleus using quantitative susceptibility mapping. Journal of Magnetic Resonance Imaging 2017, 45(5): 1352-1358

\section{ISSUE DATE:}

2017-05

\section{URL:}

http://hdl.handle.net/2433/224994

\section{RIGHT:}

This is the accepted version of the following article: [Hinoda, T., Fushimi, Y., Okada, T., Arakawa, Y., Liu, C., Yamamoto, A., Okada, T., Yoshida, K., Miyamoto, S. and Togashi, K. (2017), Quantitative assessment of gadolinium deposition in dentate nucleus using quantitative susceptibility mapping. J. Magn. Reson. Imaging, 45: 1352-1358], which has been published in final form at http://dx.doi.org/10.1002/jmri.25490. This article may be used for non-commercial purposes in accordance with Wiley Terms and Conditions for Self-Archiving.; The full-text file will be made open to the public on 18 APR 2018 in accordance with publisher's 'Terms and Conditions for Self-Archiving'.; この論文は出版社版でありませ ん。引用の際には出版社版をご確認ご利用ください。; This is not the published version. Please cite only the published version. 
Title

Quantitative Assessment of Gadolinium Deposition in Dentate Nucleus using Quantitative Susceptibility Mapping.

Authors

Takuya Hinoda, MD ${ }^{1}$, Yasutaka Fushimi, MD, PhD ${ }^{1}$,

Tomohisa Okada, $\mathrm{MD}, \mathrm{PhD}^{2}$, Yoshiki Arakawa, $\mathrm{MD}, \mathrm{PhD}^{3}$,

Chunlei Liu, PhD ${ }^{4}$, Akira Yamamoto, MD, PhD ${ }^{1}$, Tsutomu Okada, MD, PhD ${ }^{1}$, Kazumichi Yoshida, MD, $\mathrm{PhD}^{3}$, Susumu Miyamoto, $\mathrm{MD}, \mathrm{PhD}^{3}$, and Kaori Togashi, MD, PhD ${ }^{1}$.

${ }^{1}$ Department of Diagnostic Imaging and Nuclear Medicine, Kyoto University Graduate School of Medicine. 54 Shogoin Kawaharacho, Sakyoku, Kyoto, JAPAN 6068507

${ }^{2}$ Human Brain Research Center, Kyoto University Graduate School of Medicine. 54 Shogoin Kawaharacho, Sakyoku, Kyoto, JAPAN 6068507

3 Department of Neurosurgery, Kyoto University Graduate School of Medicine. 54 Shogoin Kawaharacho, Sakyoku, Kyoto, JAPAN 6068507

${ }^{4}$ Brain Imaging and Analysis Center and Department of Radiology, Duke University Medical Center. 40 Duke Medicine Circle, Davison Room 414, Durham, NC 27710 
Yasutaka Fushimi, MD, PhD

Department of Diagnostic Imaging and Nuclear Medicine

Graduate School of Medicine, Kyoto University

54 Shogoinkawaharacho, Sakyo, Kyoto, 606-8507, Japan

Office phone: $+81-75-751-3760$

Office FAX: +81-75-771-9709

Any funding information

This work was supported by JSPS KAKENHI Grant Number 25461815.

Acknowledgments

The authors are grateful to Mr. Katsutoshi Murata, and Mr. Yuta Urushibata, Siemens Healthcare K.

K., for their useful advice.

Running Title

QSM for Gadolinium Deposition 


\section{Purpose}

Gadolinium deposition in dentate nucleus (DN) has been reported after serial administration of gadoliniumbased contrast agents (GBCAs). Gadolinium complexes have paramagnetic properties, therefore, we evaluated susceptibility changes of gadolinium deposition in dentate nucleus (DN), using quantitative susceptibility mapping (QSM) for patients after serial administration of GBCAs.

\section{Materials and Methods}

48 patients with brain tumors, who had had serial GBCA administrations (GBCA group), and 48 healthy volunteers without any history of GBCA administrations (non-GBCA group) were enrolled to this study. Susceptibility values in DN on QSM and DN-to-cerebellum signal intensity ratios on unenhanced T1-weighted images ( $\mathrm{T} 1$ ratios) on 3T were analyzed. The relationship between the number of times of GBCA administrations and susceptibility values or T1 ratios were evaluated in GBCA group.

\section{Results}

Susceptibility values at DN in GBCA group were $0.107 \pm 0.029 \mathrm{ppm}$, and significantly higher than those of non-GBCA group $(0.079 \pm 0.025 \mathrm{ppm})(P<0.0001)$. T1 ratios in DN of GBCA group was $1.059 \pm 0.070$, and also significantly higher than that of non-GBCA group $(0.993 \pm 0.016)(P<0.0001)$. Spearman rank correlation coefficient between susceptibility values and the number of times of linear GBCA administration showed a modest significant correlation $(\rho=0.45, P=0.0015)$. There is good correlation between $\mathrm{T} 1$ ratios and the number of times of linear GBCA administration as reported previously $(\rho=0.76, P<0.0001)$.

\section{Conclusion}

Susceptibility values on QSM in DN of GBCA group, after serial administration of GBCAs, were significantly higher than those of non-GBCA group. 


\section{Key words}

Magnetic Resonance Imaging;

Quantitative Susceptibility Mapping;

Gadolinium-based contrast agents

Dentate nucleus 
Text

\section{INTRODUCTION}

Gadolinium-based contrast agents (GBCAs) have been widely used for contrast-material-enhanced magnetic resonance (MR) imaging. It is well known that the blood brain barrier (BBB) failure leads to the leakage of the GBCAs and high signal intensity on T1-weighted images in the central nervous system (CNS), GBCAs have been frequently used for evaluation of the central nervous system diseases such as brain tumors and demyelinating diseases. In addition to the alert of nephrogenic systemic fibrosis (NSF) due to free gadolinium deposition to cutaneous tissues, gadolinium accumulation in the dentate nucleus (DN) has gained attention after recent reports of Kanda, et al, ${ }^{1-3}$ Errante, et al, ${ }^{4}$ McDonald, et al, ${ }^{5}$ Radbruch, et al, ${ }^{6}$ Quattrocchi, et al, ${ }^{7,8}$ and Ramalho, et al. ${ }^{9}$ GBCAs are synthesized as complexes, using linear or macrocyclic chelates, and linear GBCAs have reported to be associated with T1 hyperintensity in DN. ${ }^{3}$ Most recent studies have focused T1 shortening effect of gadolinium in the DN on T1-weighted images qualitatively, ${ }^{2-6,9}$ however, susceptibility changes on quantitative susceptibility mapping (QSM) due to paramagnetic effect of gadolinium may provide a more quantitative assessment.

Magnetic susceptibility is conventionally evaluated on T2*-weighted imaging qualitatively on $\mathrm{MR},{ }^{10}$ but QSM is a robust technique due to its direct measurement of voxel-wise tissue magnetic susceptibility from phase images. ${ }^{11-15}$ Based on recent studies, QSM can provide in vivo quantitative susceptibility values. ${ }^{16,17}$ QSM has been applied to study hemorrhages, ${ }^{18,19}$ multiple sclerosis, ${ }^{20,21}$ and neurodegenerative diseases such as Alzheimer disease ${ }^{22}$ and Parkinson disease. ${ }^{23,24}$ In these diseases, iron accumulation or demyelination is supposed to occur in brain, which consequently increase magnetic susceptibility values. ${ }^{18-21,25}$ 
In this study, we hypothesized that serial usage of GBCA will increase magnetic susceptibility values in DN of humans on QSM images. Serial usage of GBCA was performed for limited purposes such as followup studies of patients with brain tumors and multiple sclerosis. Therefore, we retrospectively investigated susceptibility values in DN of brain tumor patients who had serial GBCA administration in comparison with healthy volunteers who had no history of GBCA administration

\section{MATERIALS AND METHODS}

\section{Subjects}

This single-center, retrospective observational study was performed after institutional review board approval, with waiver of the informed consent requirement. We assessed 53 consecutive patients with brain tumors since April 2014, whose original phase images of three-dimensional (3D) gradient-echo sequence were archived . 5 patients were excluded because of whole brain radiotherapy, or tumor-selective radiation therapy that included the DN within the radiation field, renal dysfunction. In total, 48 patients were finally enrolled in this study (GBCA group). Most patients underwent several MR scans with GBCA administration (linear and/or macrocyclic GBCA). The number of GBCA administration including linear and macrocyclic GBCA were abstracted from medical charts. GBCA of 0.1 mmol per kilogram of the patient's body weight was injected for each scan. Forty-eight healthy volunteers without any history of GBCA-administration were recruited in order to for a comparison group for QSM, with IRB approval and written informed consent (non-GBCA group).

\section{Images acquisition}

All the subjects had MRI examinations on 3T MR scanners (Magnetom Skyra or Trio, Siemens, Erlangen, Germany) with a 32-channel head coil. Our standard MR imaging protocol included a three- 
dimensional (3D) axial gradient-echo sequence (repetition time (TR) / echo time (TE) / echo spacing ( $\Delta \mathrm{TE}$ ), $55 \mathrm{~ms} / 3.6$ - $45.0 \mathrm{~ms} / 5.91 \mathrm{~ms}$; field of view (FOV), $240 \times 240 \mathrm{~mm}$; resolution, $0.9 \times 0.9 \times 2.0 \mathrm{~mm}$ ), a T2weighted spin-echo sequence (TR/TE, 3200/79 ms; FOV, $185 \times 220 \mathrm{~mm}$; resolution, $0.5 \times 0.5 \times 3.0 \mathrm{~mm}$ ), a fluid-attenuated inversion-recovery sequence (TR/TE, 12000/100 ms; inversion time (TI), 100 ms; FOV, 178 $\times 220 \mathrm{~mm}$; resolution, $0.7 \times 0.7 \times 3.0 \mathrm{~mm}$ ), and a 3D sagittal T1-weighted sequence (volume-interpolated breath-hold examination, or VIBE: TR/TE, 6.0 ms/2.29 ms; flip angle, $15^{\circ}$; FOV, $230 \times 230 \mathrm{~mm}$; resolution, $0.9 \times 0.9 \times 0.9 \mathrm{~mm}$, or magnetization-prepared rapid gradient-echo, or MPRAGE: TR/TE/TI, 1900 ms /2.58 ms /900 ms; flip angle, $9^{\circ}$; FOV, $230 \times 230$ mm; resolution, $0.9 \times 0.9 \times 0.9 \mathrm{~mm}$ ). 3D sagittal T1-weighted images were reformatted to axial planes parallel to the axial images.

\section{QSM analysis}

QSM calculation was conducted from the magnitude and phase images of the gradient echo images by using STI Suite version 2.10 (http://people.duke.edu/ cl160/). We first performed phase unwrapping and background phase removal using the sophisticated harmonic artifact reduction for phase data with a variable radius of the spherical kernel at the brain boundary (V-SHARP method). ${ }^{26,27}$ During this process, binary brain masks, created from the magnitude image by BET tool, were applied to facilitate the removal of background phase. ${ }^{28}$ After background phase removal, a susceptibility map was reconstructed from the resulting local tissue phase image by solving an inverse problem using the algorithm for sparse linear equations and sparse least squares (iLSQR method). ${ }^{26}$

\section{ROI analysis}

A region of interest (ROI) study was performed by two board certified neuroradiologists (T.H., 8 years of experience, Y.F., 18 years of experience) by manual placement of ROIs of DN and white matter in 
the right cerebellar hemisphere on both T1-weigheted image and QSM image using the ImageJ software

(National Institutes of Health, Bethesda, MD) (Fig. 1). ${ }^{2,3}$ ROI placement was agreed on by consensus, and ROI measurement was conducted once. In general, QSM images delineate DN very well in both groups, however, it is difficult to recognize DN on T1-weighted images for non-GBCA group, therefore, ROIs were placed by referring to QSM images. The mean of the susceptibility values of DN were measured and T1 ratios were defined as follows: mean values of DN divided by those of the cerebellar white matter on T1-weighted images.

\section{Statistics}

We performed an unpaired $t$ test with the Welch correlation to assess the difference of susceptibility values and T1 ratios of DN between the followings: (i) GBCA group (patients) vs. non-GBCA group (healthy volunteers), (ii) only macrocyclic GBCA administration in GBCA group vs. non-GBCA group, (iii) only linear GBCA or both linear and macrocyclic GBCA administrations in GBCA group vs. non-GBCA group. Nonparametric Spearman rank correlation coefficient was analyzed for the followings: (i) susceptibility values or $\mathrm{T} 1$ ratios vs. the number of times of linear GBCA administration, (ii) susceptibility values and T1 ratios in non-GBCA group vs. subjects’ age. All the statistical analyses were conducted using MedCalc version 13.3 (MedCalc Software bvba, Ostend, Belgium). $P$ values of less than .05 indicated a statistically significant difference. In the first place, multiple regression analysis was conducted with the following variables concerning the effect on the susceptibility values: the times of linear GBCA administrations, mean intervals, median intervals, last intervals, all periods. None of these variables except the times of the linear GBCA administrations $(\mathrm{P}=$ 0.0066), therefore, in the next step, we adopted Spearman Rank correlation coefficient between susceptibility values and the number of times of linear GBCA administration. 


\section{RESULTS}

Subjects

The subjects’ characteristics were shown in Table 1. The age distribution between GBCA group and non-GBCA group was not significantly different $(P=0.382)$. No patient received double-dose or triple-dose GBCA administration The median number of times of GBCA administration among GBCA group was 9.5 (4.75 -21, $25^{\text {th }}-75^{\text {th }}$ percentile, 1 - 62; range of the number). Both linear type and macrocyclic type GBCA had been administered to the same patients with long-term follow-up because linear GBCA had been used for most of gadolinium enhanced studies before NSF problem was publicly known. The median number of times of linear type GBCA administration was $5(1$ - 12.5, 0 - 48) $(n=41)$, and that of macrocyclic GBCAs administration was $4(1-7,0$ - 39) $(n=43)$. Among them, the number of macrocyclic GBCAs administration without any linear GBCA administration was $1(1-2,1-7)(n=7)$.

Susceptibility value and T1 ratio between GBCA and non-GBCA group

The susceptibility values at DN in GBCA group were significantly higher than those of non-GBCA group (mean \pm S.D., GBCA group, $0.107 \pm 0.029$ ppm; non-GBCA group, $0.079 \pm 0.025$ ppm, $P<0.0001$ ) (Fig. 2). T1 ratios at DN in GBCA group were also significantly higher than that in non-GBCA group (GBCA group, $1.059 \pm 0.070$; non-GBCA group, $0.993 \pm 0.016, P<0.0001)$ (Fig. 3).

Susceptibility values and $T 1$ ratios for linear GBCA administration

Among all the subjects of GBCA group and non-GBCA group $(n=48)$, Spearman rank correlation coefficient between susceptibility values and the number of times of linear GBCA administration showed a modest but significant correlation ( $\rho=0.45, P=0.0015)$ (Fig.4). On the other hand, there was a good 
correlation between T1 ratios and the number of times of linear GBCA administration $(\rho=0.76, P<0.0001)$

(Fig. 5). Spearman rank correlation coefficient analysis between susceptibility values and T1 ratios showed weak correlation $(\rho=0.53, P=0.0001)$ (Fig. 6).

Susceptibility values and T1 ratios for macrocyclic GBCA administration and linear GBCA administration.

The mean susceptibility values in the DN of the patients who had only macrocyclic GBCA administration $(\mathrm{n}=7)$ was $0.087 \pm 0.019 \mathrm{ppm}$; the mean $\mathrm{T} 1$ ratio was $0.979 \pm 0.031$. There was no significant difference of susceptibility values $(P=0.348)$ or T1 ratios $(P=0.745)$ between macrocyclic GBCA-only group non-GBCA group. The mean susceptibility value in the DN of those who had only linear GBCA ( $\mathrm{n}=$ 5) or both linear and macrocyclic GBCA administration $(n=36)$ was $0.111 \pm 0.029 \mathrm{ppm}$; the mean T1 ratio was $1.070 \pm 0.069$. There is significant difference of susceptibility values $(P<0.0001)$ and T1 ratio $(P<$ 0.0001) between linear or both linear and macrocyclic GBCA group and non-GBCA group.

Susceptibility values and T1 ratios in non-GBCA group in comparison with subjects'age.

Susceptibility values have weak correlation between subjects’ age in non-GBCA group showed weak correlation ( $\rho=0.38, P=0.0074)$, and T1 ratios have also weak negative correlation $(\rho=-0.331, P=0.0217)$.

\section{DISCUSSON}

Our analyses have shown that susceptibility values in DN of GBCA group (tumor patients group after serial GBCA administrations) were significantly higher than those of non-GBCA group (healthy controls) and T1 ratios of GBCA group are significantly higher than those of non-GBCA group. In the current study, we focused on the serial injection of GBCA and gadolinium deposition in DN as indicated by previous reports. ${ }^{1,5}$

The results of $\mathrm{T} 1$ ratios are consistent with previous reports ${ }^{1,5}$ and we again confirmed $\mathrm{T} 1$ hyperintensity due 
to gadolinium deposition after serial GBCA administrations.

DN is known to have rich iron content, ${ }^{29}$ and high susceptibility in DN has been reported. ${ }^{30}$ Gadolinium is a strong paramagnetic substance with a molar susceptibility of $325 \mathrm{ppm} \cdot \mathrm{L} / \mathrm{mol}$. Therefore, theoretically susceptibility values in DN should elevate when the T1 ratios shows gadolinium deposition in DN after serial GBCA administrations. The results of higher susceptibility values in DN of GBCA group than non-GBCA group supports our initial hypothesis that QSM can be a biomarker of gadolinium deposition in vivo in addition to T1-weghted images.

In recent study, macrocyclic GBCA shows less or no abnormal deposition in the DN than linear GBCA, which supported that macrocyclic GBCA has excellent chemical stability without dissociation of free gadolinium. ${ }^{3}$ In this study, the number of GBCA group with only macrocyclic GBCA was quite small, but the susceptibility values were close to those of non-GBCA group and had no significant difference. On the contrary, the susceptibility values of GBCA group, with previous linear GBCA administration with/without macrocyclic GBCA administration, were significantly higher than those of non-GBCA group. On T1-weighted images, previous reports have showed the strong association between the hyperintensity in the DN and linear GBCA, ${ }^{3}$ our result of this study could reinforce the previous result.

Gadolinium deposition in DN has been correlated with the number of times of linear GBCA administration as reported in the literature..$^{1-3,5}$ Our study showed that susceptibility values in DN correlated less well with the number of times of linear GBCA administration than T1 ratios did. One reason for the weaker correlation between susceptibility and the number of times of linear GBCA administration is the endogenous substances causing susceptibility changes in DN cause less T1 shortening effect and vary in individuals; in addition, the normal range of the susceptibility values in DN is quite wide and can be affected 
by the variation of age in the group. Relaxivities (R1 and R2) of GBCA is 3 - $5 \mathrm{~L} / \mathrm{mM} / \mathrm{s}$ range and T1 relaxation time of brain tissue is relatively long in contrast to T2 relaxation time of brain tissue, therefore, deposition of gadolinium will effectively cause T1 shortening effect. In spite of predominance of T1 shortening effect, significant susceptibility changes associated putative gadolinium deposition in GBCA group were demonstrated in this study. Moreover, the QSM showed wider variation in DN. Therefore this wider variation of susceptibility values in DN could be the reason for weaker correlation between susceptibility values and the number of times of GBCA administrations.

In a prior postmortem study, iron content varied with age in the globus pallidus, caudate nucleus, and putamen $^{29}$ and on MR images DN showed various signal intensity, ${ }^{31}$ specifically, susceptibility values of DN also could change due to iron deposition with aging and this have been reported in the recent study using QSM. $^{32,33}$ In our study, the result of Spearman rank correlation coefficient between susceptibility values in DN and subjects' age showed weak but significant correlation that were consistent with previous studies. ${ }^{32,33}$ Nevertheless, in a short time window (e.g. 2-3 years) when age-related changes are negligible, QSM provides a means to quantitatively monitor GBCA deposition in DN longitudinally because of its quantitative nature and high sensitivity to GBCA.

This retrospective study of QSM and gadolinium deposition in DN presents several limitations. First, to assess the deposition of gadolinium in DN correctly, sequential prospective study should be needed. In this study, consecutive evaluation of susceptibility values and T1 ratios was not performed. Macrocyclic GBCA was reported to be low gadolinium deposition in contrast to linear GBCA, and linear GBCA is avoided to use today because of the risk of NSF, therefore, it is difficult to plan a prospective comparison study between macrocyclic and linear GBCA in human subjects. Second, the number of times of GBCA administration were 
explored on medical charts as much as possible, but some patients with long-term follow-up in GBCA group might have some additional GBCA administration at outside hospitals which were not counted in number of times of GBCA administration. Third, globus pallidus was not included for evaluation in this study. Several papers revealed relatively higher signal on T1-weighted image probably due to gadolinium deposition ${ }^{2} 65$, however, the signal increase on T1-weighted image was much smaller than that of dentate nucleus. In addition, physiological iron accumulation is frequently seen in globus pallidus ${ }^{29}$, therefore, we solely focused the susceptibility change in the dentate nucleus.

In conclusion, the susceptibility values in DN after serial GBCA administrations were significantly higher in comparison with healthy volunteers. This fact was comparison with our initial hypothesis that QSM could detect the gadolinium deposition at DN after serial GBCA administrations. 


\section{REFERENCES}

1. Kanda T, Fukusato T, Matsuda M, et al. Gadolinium-based Contrast Agent Accumulates in the Brain Even in Subjects without Severe Renal Dysfunction: Evaluation of Autopsy Brain Specimens with Inductively Coupled Plasma Mass Spectroscopy. Radiology $2015 ; 276: 228-232$.

2. Kanda T, Ishii K, Kawaguchi H, Kitajima K, Takenaka D. High signal intensity in the dentate nucleus and globus pallidus on unenhanced T1-weighted MR images: relationship with increasing cumulative dose of a gadolinium-based contrast material. Radiology 2014;270:834-841.

3. Kanda T, Osawa M, Oba H, et al. High Signal Intensity in Dentate Nucleus on Unenhanced T1-weighted MR Images: Association with Linear versus Macrocyclic Gadolinium Chelate Administration. Radiology 2015;275:803-809.

4. Errante Y, Cirimele V, Mallio CA, Di Lazzaro V, Zobel BB, Quattrocchi CC. Progressive increase of T1 signal intensity of the dentate nucleus on unenhanced magnetic resonance images is associated with cumulative doses of intravenously administered gadodiamide in patients with normal renal function, suggesting dechelation. Investigative radiology 2014;49:685-690.

5. McDonald RJ, McDonald JS, Kallmes DF, et al. Intracranial Gadolinium Deposition after Contrast-enhanced MR Imaging. Radiology 2015;275:772-782.

6. Radbruch A, Weberling LD, Kieslich PJ, et al. Gadolinium retention in the dentate 
nucleus and globus pallidus is dependent on the class of contrast agent. Radiology 2015;275:783-791.

7. Quattrocchi CC, Mallio CA, Errante Y, Beomonte Zobel B. High T1 Signal Intensity in Dentate Nucleus after Multiple Injections of Linear Gadolinium Chelates. Radiology 2015;276:616-617.

8. Quattrocchi CC, Mallio CA, Errante Y, et al. Gadodiamide and Dentate Nucleus T1 Hyperintensity in Patients With Meningioma Evaluated by Multiple Follow-Up Contrast-Enhanced Magnetic Resonance Examinations With No Systemic Interval Therapy. Investigative radiology 2015;50:470-472.

9. Ramalho J, Castillo M, AlObaidy M, et al. High Signal Intensity in Globus Pallidus and Dentate Nucleus on Unenhanced T1-weighted MR Images: Evaluation of Two Linear Gadolinium-based Contrast Agents. Radiology 2015:150872.

10. Haacke EM, Xu Y, Cheng YC, Reichenbach JR. Susceptibility weighted imaging (SWI). Magnetic resonance in medicine : official journal of the Society of Magnetic Resonance in Medicine / Society of Magnetic Resonance in Medicine 2004;52:612-618.

11. de Rochefort L, Liu T, Kressler B, et al. Quantitative susceptibility map reconstruction from MR phase data using bayesian regularization: validation and application to brain imaging. Magnetic resonance in medicine : official journal of the Society of Magnetic Resonance in Medicine / Society of Magnetic Resonance in Medicine 2010;63:194-206.

12. Li W, Wu B, Liu C. Quantitative susceptibility mapping of human brain reflects spatial variation in tissue composition. NeuroImage 2011;55:1645-1656. 
13. Liu T, Liu J, de Rochefort L, et al. Morphology enabled dipole inversion (MEDI) from a single-angle acquisition: comparison with COSMOS in human brain imaging. Magnetic resonance in medicine: official journal of the Society of Magnetic Resonance in Medicine / Society of Magnetic Resonance in Medicine 2011;66:777-783.

14. Liu T, Spincemaille P, de Rochefort L, Kressler B, Wang Y. Calculation of susceptibility through multiple orientation sampling (COSMOS): a method for conditioning the inverse problem from measured magnetic field map to susceptibility source image in MRI. Magnetic resonance in medicine : official journal of the Society of Magnetic Resonance in Medicine / Society of Magnetic Resonance in Medicine 2009;61:196-204.

15. Schweser F, Deistung A, Lehr BW, Reichenbach JR. Quantitative imaging of intrinsic magnetic tissue properties using MRI signal phase: an approach to in vivo brain iron metabolism? NeuroImage 2011;54:2789-2807.

16. Hinoda T, Fushimi Y, Okada T, et al. Quantitative Susceptibility Mapping at 3 T and 1.5 T: Evaluation of Consistency and Reproducibility. Investigative radiology 2015;50:522530.

17. Deh K, Nguyen TD, Eskreis-Winkler S, et al. Reproducibility of quantitative susceptibility mapping in the brain at two field strengths from two vendors. Journal of magnetic resonance imaging : JMRI 2015.

18. Chen W, Zhu W, Kovanlikaya I, et al. Intracranial calcifications and hemorrhages: characterization with quantitative susceptibility mapping. Radiology 2014;270:496-505.

19. Liu T, Surapaneni K, Lou M, Cheng L, Spincemaille P, Wang Y. Cerebral microbleeds: 
burden assessment by using quantitative susceptibility mapping. Radiology 2012;262:269-278.

20. Langkammer C, Liu T, Khalil M, et al. Quantitative susceptibility mapping in multiple sclerosis. Radiology 2013;267:551-559.

21. Chen W, Gauthier SA, Gupta A, et al. Quantitative susceptibility mapping of multiple sclerosis lesions at various ages. Radiology 2014;271:183-192.

22. Acosta-Cabronero J, Williams GB, Cardenas-Blanco A, Arnold RJ, Lupson V, Nestor PJ. In vivo quantitative susceptibility mapping (QSM) in Alzheimer's disease. PloS one 2013;8:e81093.

23. Barbosa JH, Santos AC, Tumas V, et al. Quantifying brain iron deposition in patients with Parkinson's disease using quantitative susceptibility mapping, R2 and R2. Magnetic resonance imaging 2015;33:559-565.

24. Ide S, Kakeda S, Ueda I, et al. Internal structures of the globus pallidus in patients with Parkinson's disease: evaluation with quantitative susceptibility mapping (QSM). Eur Radiol 2015;25:710-718.

25. Lotfipour AK, Wharton S, Schwarz ST, et al. High resolution magnetic susceptibility mapping of the substantia nigra in Parkinson's disease. Journal of magnetic resonance imaging : JMRI 2012;35:48-55.

26. Li W, Avram AV, Wu B, Xiao X, Liu C. Integrated Laplacian-based phase unwrapping and background phase removal for quantitative susceptibility mapping. NMR in biomedicine $2014 ; 27: 219-227$. 
27. $\mathrm{Wu}$ B, Li W, Guidon A, Liu C. Whole brain susceptibility mapping using compressed sensing. Magnetic resonance in medicine : official journal of the Society of Magnetic Resonance in Medicine / Society of Magnetic Resonance in Medicine 2012;67:137-147.

28. Smith SM. Fast robust automated brain extraction. Human brain mapping 2002;17:143155.

29. Hallgren B, Sourander P. The effect of age on the non-haemin iron in the human brain. Journal of neurochemistry 1958;3:41-51.

30. Langkammer C, Schweser F, Krebs N, et al. Quantitative susceptibility mapping (QSM) as a means to measure brain iron? A post mortem validation study. NeuroImage 2012;62:1593-1599.

31. Thomas LO, Boyko OB, Anthony DC, Burger PC. MR detection of brain iron. AJNR American journal of neuroradiology 1993;14:1043-1048.

32. Liu M, Liu S, Ghassaban K, et al. Assessing global and regional iron content in deep gray matter as a function of age using susceptibility mapping. Journal of magnetic resonance imaging : JMRI 2015.

33. Li W, Wu B, Batrachenko A, et al. Differential developmental trajectories of magnetic susceptibility in human brain gray and white matter over the lifespan. Human brain mapping 2014;35:2698-2713. 
TABLE 1

\section{Subjects Characteristics}

Parameter

Total no. of subjects

Age (years \pm standard deviation)

$\operatorname{Sex}(M: F)$

History of brain surgery

History of chemotherapy

History of radiation therapy

Diagnosis

High grade glioma

Low grade glioma

Tumors other than glioma

No. of contrast-enhanced MR imaging examination

No. of linear GBCAs administration

No. of macrocyclic GBCAs administration

GBCA group

48

$49.6 \pm 15.1^{*}$

(23: 25)

42

29

28

18

15

$15^{* *}$
non-GBCA group

48

$46.3 \pm 21.9 *$

(32: 16)

\section{0}

0

0

0

0

0

* Mean \pm standard deviation

** Meningioma 8, hemangiopericytoma 1, PPTID 1, dermoid 1, colloid 1, cavernous angioma 2, schwannoma

1.

*** Median (25th-75th percentile)

Note that No. represents the number of times. The radiation field did not cover the cerebellum in radiation

therapy. 


\section{FIGURE LEGENDS}

\section{Figure 1.}

Axial images in a 71-year-old woman with left-temporal-lobe glioblastoma who had underwent seven times of macrocyclic GBCA and 22 times of linear GBCA administrations (a, b). DN showed a high susceptibility value on QSM (a) and hyperintensity on T1-weighted images (b). Axial images in a 30-year-old man with right-frontal-lobe glioblastoma who had underwent eleven times of macrocyclic GBCA and 31 times of linear GBCA administrations (c, d). DN showed a high susceptibility value on QSM (c) and hyperintensity on T1weighted images (d). Axial images in a 28-year-old man without any GBCA administration (e, f). The DN showed a less susceptibility value on QSM (e) and did not show hyperintensity on T1-weighted images (f).

\section{Figure 2.}

Graph of the mean susceptibility value difference between GBCA group, who had had serial GBCA administrations, and non-GBCA group, who had had no GBCA administration. Susceptibility values at DN in GBCA group were significantly higher than those in non-GBCA group $(P<0.0001)$. Note that error bars represent the $95 \%$ confidence intervals.

\section{Figure 3.}

Graph of the mean T1 ratio (signal ratio of dentate nucleus/cerebellum cortex on T1-weighted images) differences between GBCA group and non-GBCA group. T1 ratios in GBCA group were also significantly higher than those in non-GBCA group $(P<0.0001)$. Note that error bars represent the 95\% confidence 
intervals.

\section{Figure 4.}

Graph shows result of scattered plots and Spearman rank correlation coefficient between susceptibility values at DN in GBCA group and linear GBCA administrations.

\section{Figure 5.}

Graph shows result of scattered plots and Spearman rank correlation coefficient between T1 ratios of DN on

T1-weighted images in GBCA group and the number of linear GBCA administrations.

\section{Figure 6.}

Graph shows result of scattered plots and Spearman rank correlation coefficient between T1 ratios and susceptibility values at DN. 

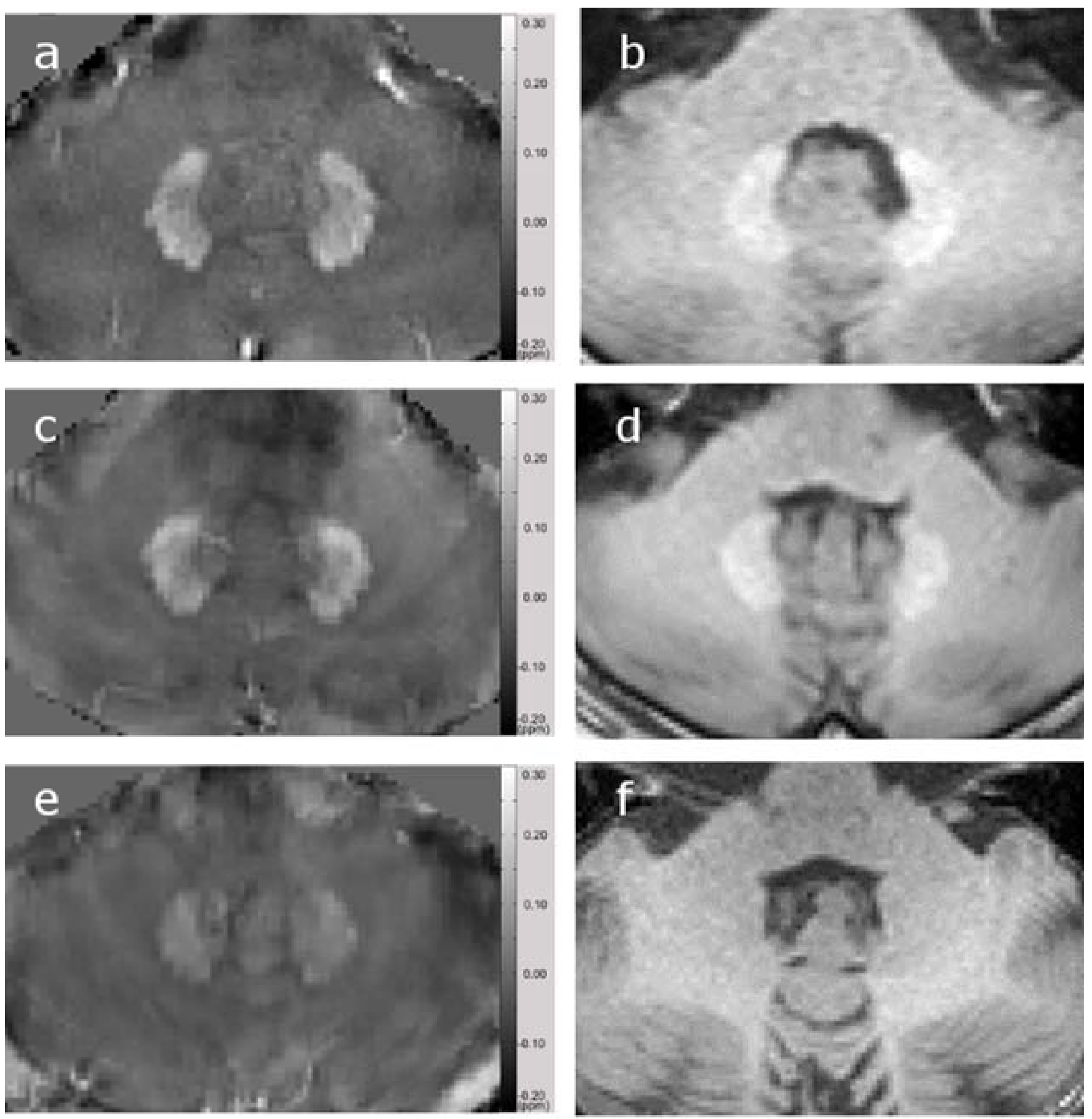

Figure 1 


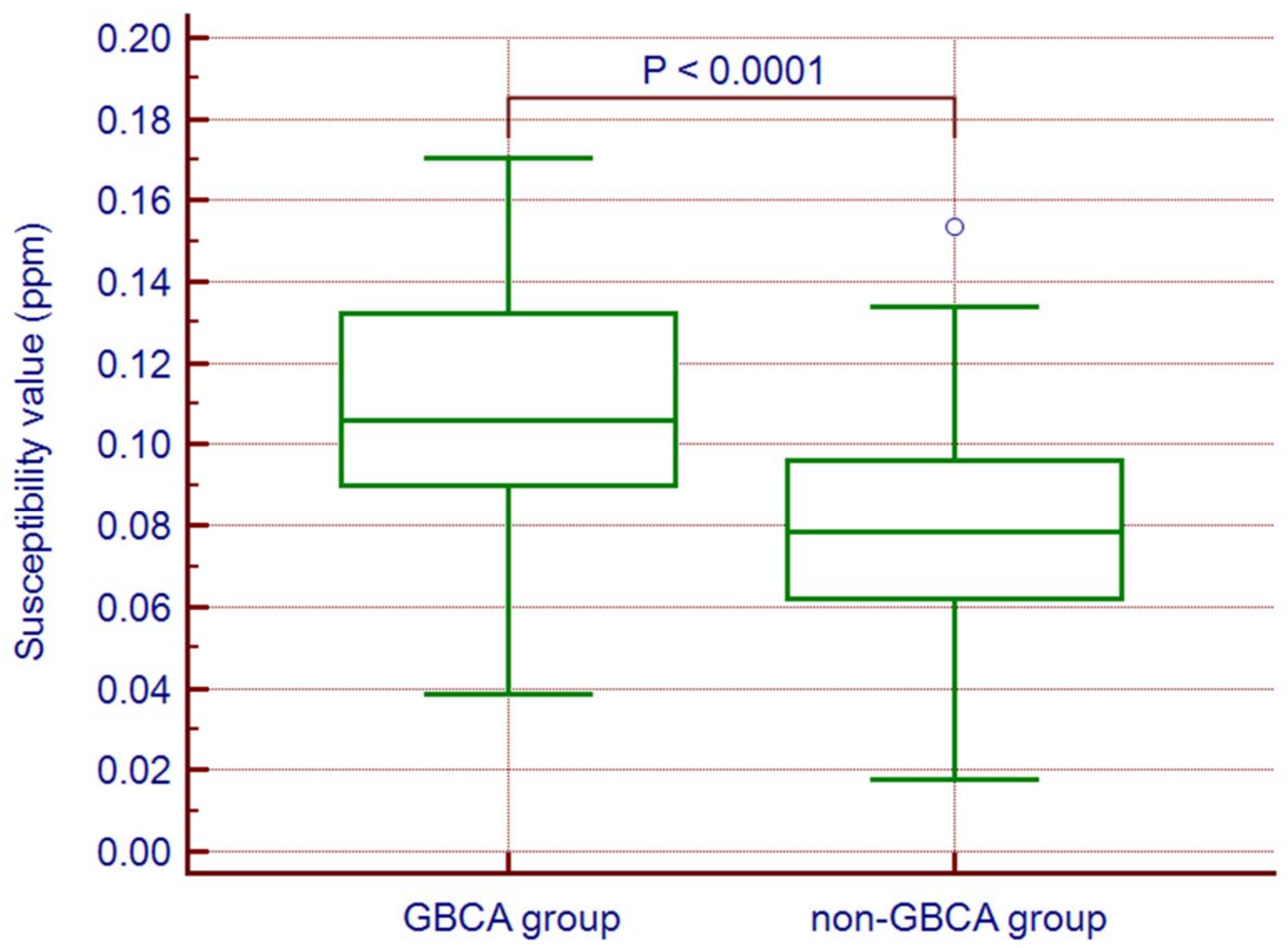

Figure 2 


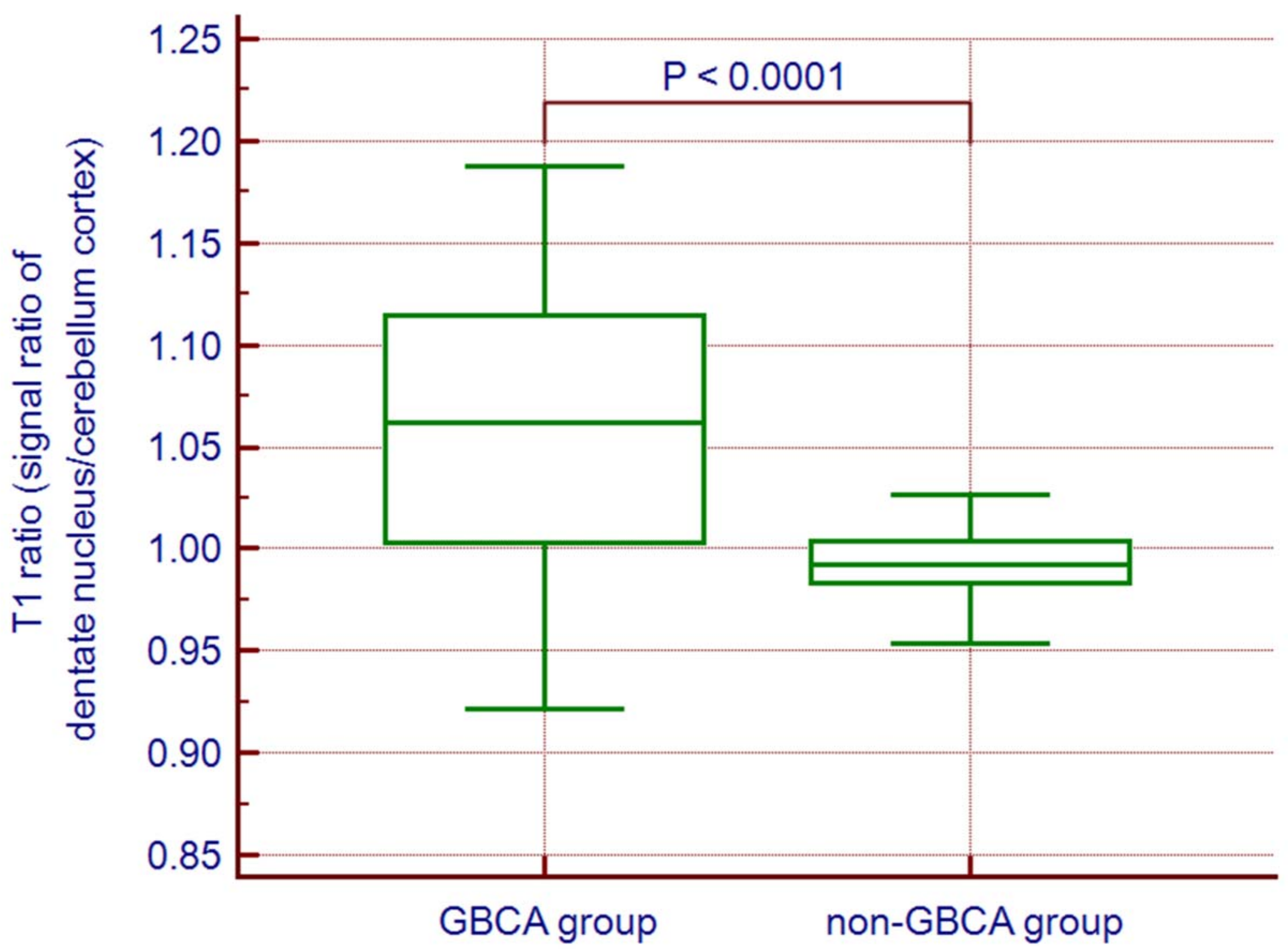

Figure 3 


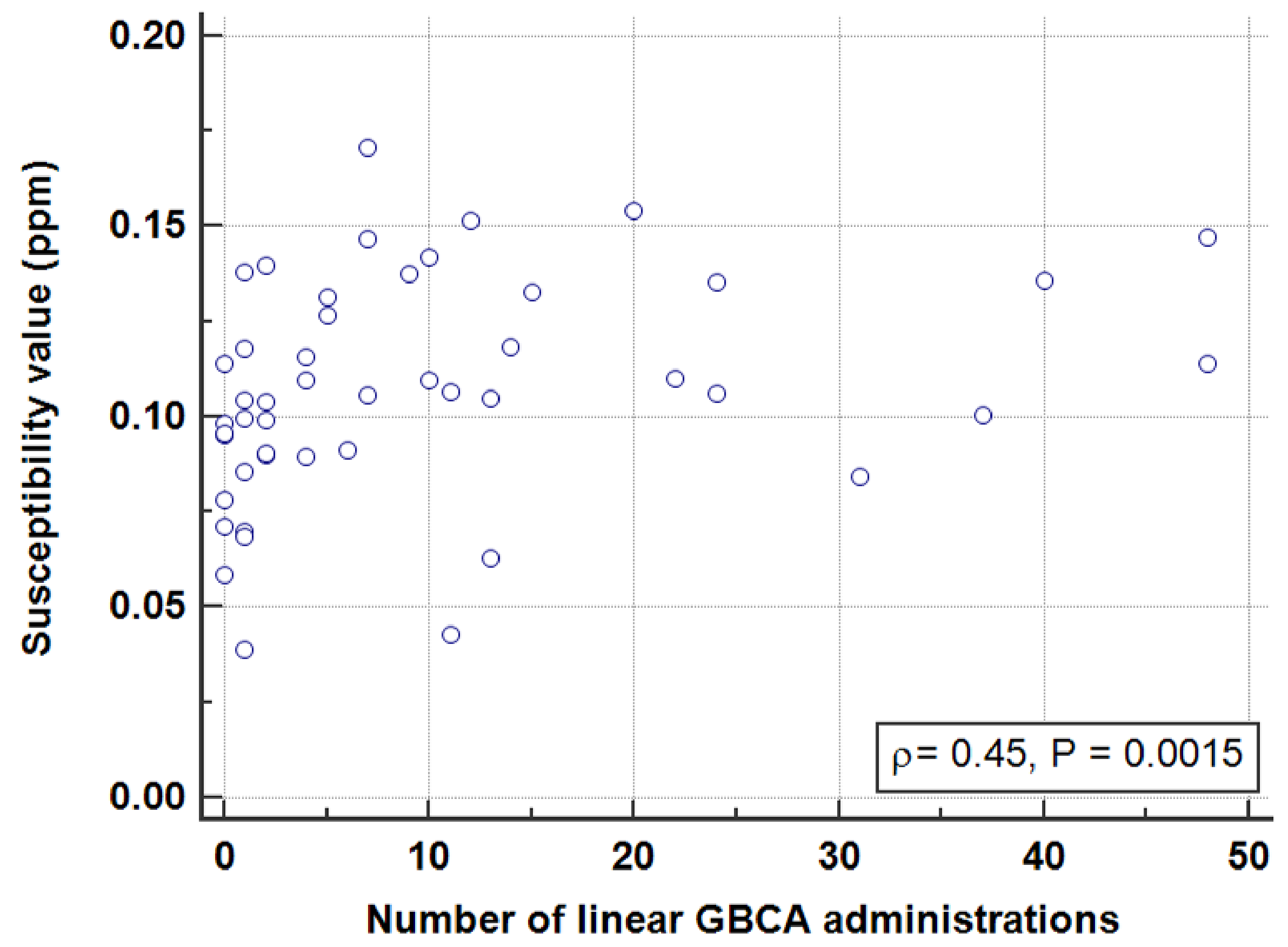




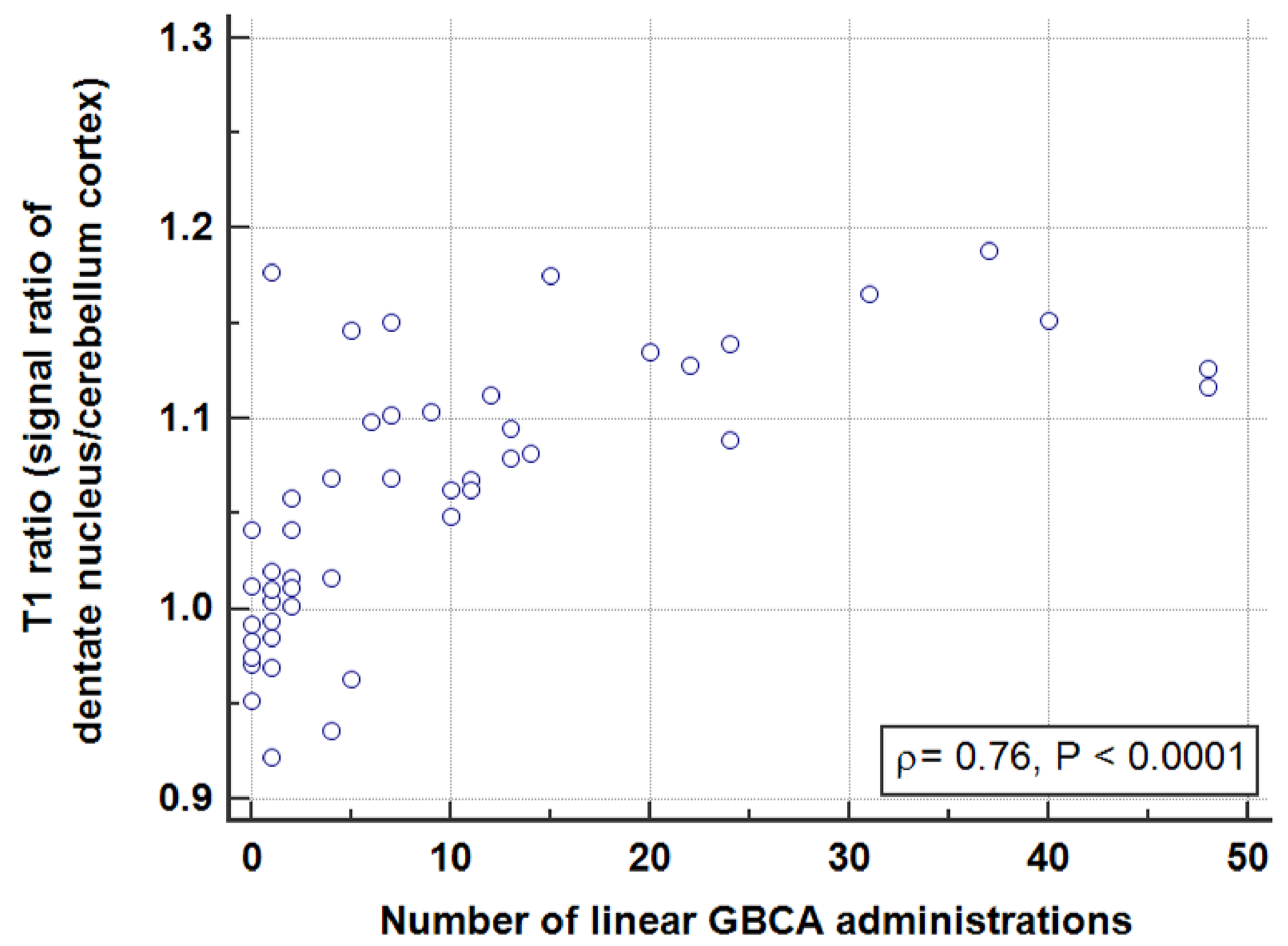

Figure 5 


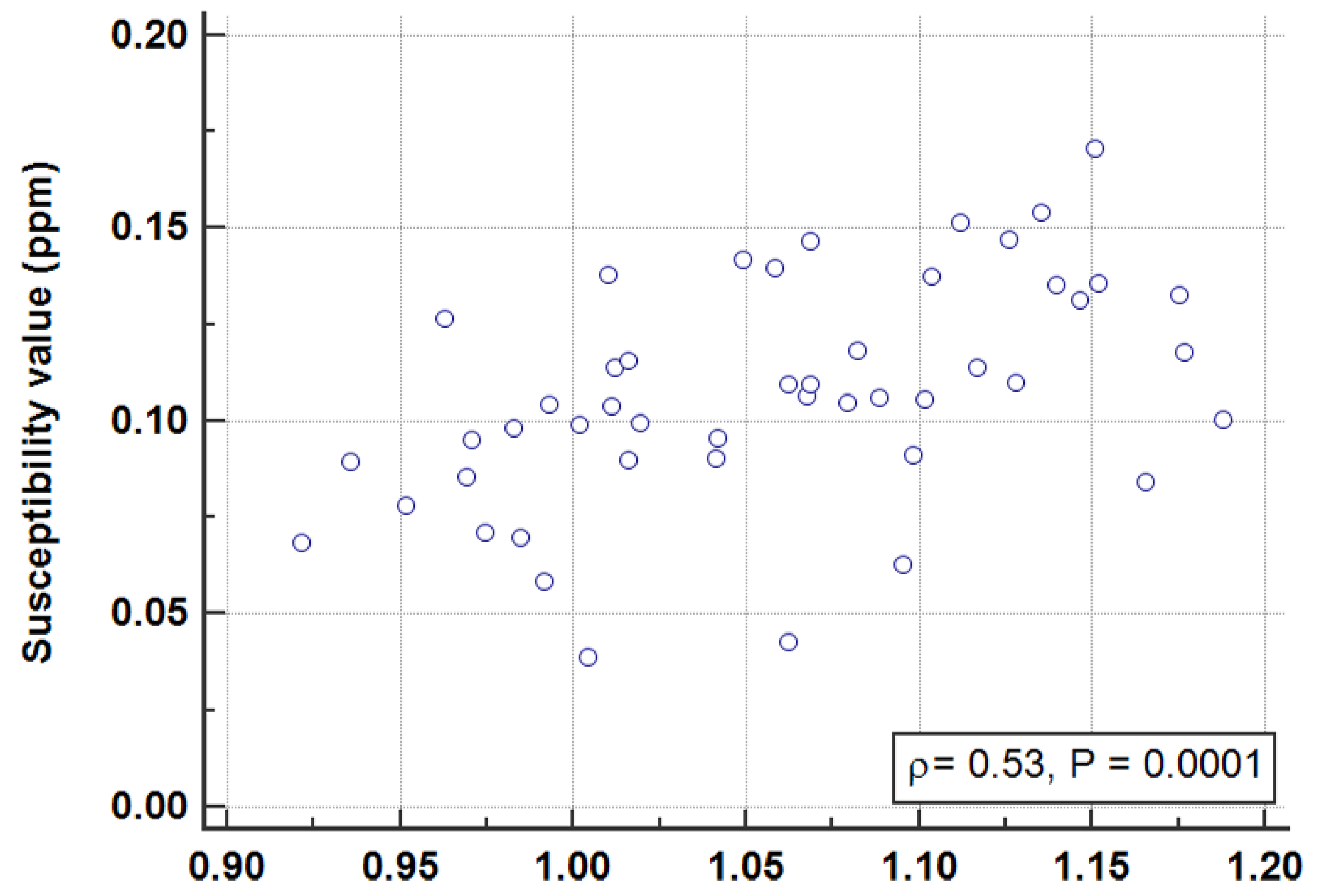

T1 ratio (signal ratio of dentate nucleus/cerebellum cortex) 